Commun. Korean Math. Soc. 27 (2012), No. 3, pp. 591-602

http://dx.doi.org/10.4134/CKMS.2012.27.3.591

\title{
INTEGRABILITY OF DISTRIBUTIONS IN GCR-LIGHTLIKE SUBMANIFOLDS OF INDEFINITE KAEHLER MANIFOLDS
}

\author{
Rakesh Kumar, Sangeet Kumar, and Rakesh Kumar Nagaich
}

ABSTRACT. In present paper we establish conditions for the integrability of various distributions of $G C R$-lightlike submanifolds and obtain conditions for the distributions to define totally geodesic foliations in $G C R$ lightlike submanifolds.

\section{Introduction}

The study of geometry of Cauchy-Riemann $(C R)$-submanifolds in Kaehler manifolds was initiated by Bejancu [2], which include holomorphic and totally real submanifolds as special cases and has been further studied by Bejancu $[3,4]$, Chen [6], Duggal [7, 8], Yano and Kon [17, 18] and others.

The geometry of lightlike submanifolds was initiated by Kupeli and further developed by Bejancu and Duggal [9] and they also introduced the notion of $C R$-lightlike submanifolds of indefinite Kaehler manifolds. But this class of submanifolds exclude the complex and totally real submanifolds as subcases. Later on, Duggal and Sahin [11] introduced $S C R$-lightlike submanifolds of indefinite Kaehler manifolds. Since there was no inclusion relation between $C R$ and $S C R$ cases therefore Duggal and Sahin [12] introduced a new class called $G C R$-lightlike submanifolds of indefinite Kaehler manifolds which is an umbrella for all these types of submanifolds. In present paper we establish conditions for the integrability of various distributions of $G C R$-lightlike submanifolds and obtain conditions for the distributions to define totally geodesic foliations in $G C R$-lightlike submanifolds.

\section{Lightlike submanifolds}

We recall notations and fundamental equations for lightlike submanifolds, which are due to the book [9] by Duggal and Bejancu.

Let $(\bar{M}, \bar{g})$ be a real $(m+n)$-dimensional semi-Riemannian manifold of constant index $q$ such that $m, n \geq 1,1 \leq q \leq m+n-1$ and $(M, g)$ be an

Received May 9, 2011.

2010 Mathematics Subject Classification. 53C15, 53C40, 53C50.

Key words and phrases. indefinite Kaehler manifold, GCR-lightlike submanifold, integrability of distributions. 
$m$-dimensional submanifold of $\bar{M}$ and $g$ the induced metric of $\bar{g}$ on $M$. If $\bar{g}$ is degenerate on the tangent bundle $T M$ of $M$, then $M$ is called a lightlike submanifold of $\bar{M}$. For a degenerate metric $g$ on $M$

$$
T M^{\perp}=\cup\left\{u \in T_{x} \bar{M}: \bar{g}(u, v)=0, \forall v \in T_{x} M, x \in M\right\},
$$

is a degenerate $n$-dimensional subspace of $T_{x} \bar{M}$. Thus both $T_{x} M$ and $T_{x} M^{\perp}$ are degenerate orthogonal subspaces but no longer complementary. In this case, there exists a subspace $\operatorname{Rad} T_{x} M=T_{x} M \cap T_{x} M^{\perp}$ which is known as radical (null) subspace. If the mapping

$$
\operatorname{Rad} T M: x \in M \longrightarrow \operatorname{Rad} T_{x} M,
$$

defines a smooth distribution on $M$ of rank $r>0$, then the submanifold $M$ of $\bar{M}$ is called an $r$-lightlike submanifold and $\operatorname{Rad} T M$ is called the radical distribution on $M$.

Screen distribution $S(T M)$ is a semi-Riemannian complementary distribution of $\operatorname{Rad}(T M)$ in $T M$, that is

$$
T M=\operatorname{Rad} T M \perp S(T M)
$$

and $S\left(T M^{\perp}\right)$ is a complementary vector subbundle to $\operatorname{Rad} T M$ in $T M^{\perp}$. Let $\operatorname{tr}(T M)$ and $\operatorname{ltr}(T M)$ be complementary (but not orthogonal) vector bundles to $T M$ in $\left.T \bar{M}\right|_{M}$ and to $\operatorname{Rad} T M$ in $S\left(T M^{\perp}\right)^{\perp}$ respectively. Then we have

$$
\operatorname{tr}(T M)=\operatorname{ltr}(T M) \perp S\left(T M^{\perp}\right),
$$

(5) $\left.T \bar{M}\right|_{M}=T M \oplus \operatorname{tr}(T M)=(\operatorname{Rad} T M \oplus \operatorname{ltr}(T M)) \perp S(T M) \perp S\left(T M^{\perp}\right)$.

Let $u$ be a local coordinate neighborhood of $M$ and consider the local quasiorthonormal fields of frames of $\bar{M}$ along $M$, on $u$ as $\left\{\xi_{1}, \ldots, \xi_{r}, W_{r+1}, \ldots, W_{n}\right.$, $\left.N_{1}, \ldots, N_{r}, X_{r+1}, \ldots, X_{m}\right\}$, where $\left\{\xi_{1}, \ldots, \xi_{r}\right\},\left\{N_{1}, \ldots, N_{r}\right\}$ are local lightlike bases of $\Gamma\left(\left.\operatorname{Rad} T M\right|_{u}\right), \Gamma\left(\left.\operatorname{ltr}(T M)\right|_{u}\right)$ and $\left\{W_{r+1}, \ldots, W_{n}\right\},\left\{X_{r+1}, \ldots, X_{m}\right\}$ are local orthonormal bases of $\Gamma\left(\left.S\left(T M^{\perp}\right)\right|_{u}\right)$ and $\Gamma\left(\left.S(T M)\right|_{u}\right)$ respectively. For this quasi-orthonormal fields of frames, we have:

Theorem $2.1([9])$. Let $\left(M, g, S(T M), S\left(T M^{\perp}\right)\right)$ be an $r$-lightlike submanifold of a semi-Riemannian manifold $(\bar{M}, \bar{g})$. Then there exists a complementary vector bundle $\operatorname{ltr}(T M)$ of $\operatorname{Rad} T M$ in $S\left(T M^{\perp}\right)^{\perp}$ and a basis of $\Gamma\left(\left.\operatorname{ltr}(T M)\right|_{u}\right)$ consisting of smooth section $\left\{N_{i}\right\}$ of $\left.S\left(T M^{\perp}\right)^{\perp}\right|_{u}$, where $u$ is a coordinate neighborhood of $M$ such that

$$
\bar{g}\left(N_{i}, \xi_{j}\right)=\delta_{i j}, \quad \bar{g}\left(N_{i}, N_{j}\right)=0 \text { for any } i, j \in\{1,2, \ldots, r\},
$$

where $\left\{\xi_{1}, \ldots, \xi_{r}\right\}$ is a lightlike basis of $\Gamma(\operatorname{Rad}(T M))$.

Let $\bar{\nabla}$ be the Levi-Civita connection on $\bar{M}$ then according to the decomposition (5), the Gauss and Weingarten formulas are given by

$$
\begin{gathered}
\bar{\nabla}_{X} Y=\nabla_{X} Y+h(X, Y), \quad \forall X, Y \in \Gamma(T M), \\
\bar{\nabla}_{X} U=-A_{U} X+\nabla_{X}^{\perp} U, \quad \forall X \in \Gamma(T M), U \in \Gamma(\operatorname{tr}(T M)),
\end{gathered}
$$


where $\left\{\nabla_{X} Y, A_{U} X\right\}$ and $\left\{h(X, Y), \nabla_{X}^{\frac{1}{}} U\right\}$ belong to $\Gamma(T M)$ and $\Gamma(\operatorname{tr}(T M))$, respectively. Here $\nabla$ is a torsion-free linear connection on $M, h$ is a symmetric bilinear form on $\Gamma(T M)$ which is called second fundamental form, $A_{U}$ is a linear operator on $M$ and known as shape operator.

According to (4), considering the projection morphisms $L$ and $S$ of $\operatorname{tr}(T M)$ on $\operatorname{ltr}(T M)$ and $S\left(T M^{\perp}\right)$, respectively, then (7) and (8) become

$$
\begin{gathered}
\bar{\nabla}_{X} Y=\nabla_{X} Y+h^{l}(X, Y)+h^{s}(X, Y), \\
\bar{\nabla}_{X} U=-A_{U} X+D_{X}^{l} U+D_{X}^{s} U,
\end{gathered}
$$

where we put $h^{l}(X, Y)=L(h(X, Y)), h^{s}(X, Y)=S(h(X, Y)), D_{X}^{l} U=L\left(\nabla_{X}^{\frac{1}{X}} U\right)$, $D_{X}^{s} U=S\left(\nabla \frac{1}{X} U\right)$.

As $h^{l}$ and $h^{s}$ are $\Gamma(\operatorname{ltr}(T M))$-valued and $\Gamma\left(S\left(T M^{\perp}\right)\right)$-valued respectively, therefore they are called as the lightlike second fundamental form and the screen second fundamental form on $M$. In particular

$$
\begin{aligned}
& \bar{\nabla}_{X} N=-A_{N} X+\nabla_{X}^{l} N+D^{s}(X, N), \\
& \bar{\nabla}_{X} W=-A_{W} X+\nabla_{X}^{s} W+D^{l}(X, W),
\end{aligned}
$$

where $X \in \Gamma(T M), N \in \Gamma(\operatorname{ltr}(T M))$ and $W \in \Gamma\left(S\left(T M^{\perp}\right)\right)$. Using (9)-(12) we obtain

$$
\begin{gathered}
\bar{g}\left(h^{s}(X, Y), W\right)+\bar{g}\left(Y, D^{l}(X, W)\right)=g\left(A_{W} X, Y\right), \\
\bar{g}\left(h^{l}(X, Y), \xi\right)+\bar{g}\left(Y, h^{l}(X, \xi)\right)+g\left(Y, \nabla_{X} \xi\right)=0, \\
\bar{g}\left(A_{N} X, N^{\prime}\right)+\bar{g}\left(N, A_{N^{\prime}} X\right)=0
\end{gathered}
$$

for any $\xi \in \Gamma(\operatorname{Rad} T M), W \in \Gamma\left(S\left(T M^{\perp}\right)\right)$ and $N, N^{\prime} \in \Gamma(\operatorname{ltr}(T M))$.

Let $P$ be the projection morphism of $T M$ on $S(T M)$ then using (3), we can induce some new geometric objects on the screen distribution $S(T M)$ on $M$ as

$$
\begin{gathered}
\nabla_{X} P Y=\nabla_{X}^{*} P Y+h^{*}(X, P Y), \\
\nabla_{X} \xi=-A_{\xi}^{*} X+\nabla_{X}^{* t} \xi
\end{gathered}
$$

for any $X, Y \in \Gamma(T M)$ and $\xi \in \Gamma(\operatorname{Rad} T M)$, where $\left\{\nabla_{X}^{*} P Y, A_{\xi}^{*} X\right\}$ and $\left\{h^{*}(X\right.$, $\left.P Y), \nabla_{X}^{* t} \xi\right\}$ belong to $\Gamma(S(T M))$ and $\Gamma(\operatorname{Rad} T M)$, respectively. $\nabla^{*}$ and $\nabla^{* t}$ are linear connections on complementary distributions $S(T M)$ and $\operatorname{Rad} T M$, respectively. $h^{*}$ and $A^{*}$ are $\Gamma(\operatorname{Rad} T M)$-valued and $\Gamma(S(T M))$-valued bilinear forms and called as the second fundamental forms of distributions $S(T M)$ and $\operatorname{Rad} T M$, respectively.

Using (9), (10), (16) and (17), we obtain

$$
\begin{gathered}
\bar{g}\left(h^{l}(X, P Y), \xi\right)=g\left(A_{\xi}^{*} X, P Y\right), \\
\bar{g}\left(h^{*}(X, P Y), N\right)=\bar{g}\left(A_{N} X, P Y\right)
\end{gathered}
$$


for any $X, Y \in \Gamma(T M), \xi \in \Gamma(\operatorname{Rad}(T M))$ and $N \in \Gamma(\operatorname{ltr}(T M))$.

From the geometry of Riemannian submanifolds and non degenerate submanifolds, it is known that the induced connection $\nabla$ on a non degenerate submanifold is a metric connection. Unfortunately, this is not true for a lightlike submanifold. Indeed, considering $\bar{\nabla}$ a metric connection then we have

$$
\left(\nabla_{X} g\right)(Y, Z)=\bar{g}\left(h^{l}(X, Y), Z\right)+\bar{g}\left(h^{l}(X, Z), Y\right)
$$

for any $X, Y, Z \in \Gamma(T M)$. From [9] page 171, using the properties of linear connections we have

$$
\begin{aligned}
& \left(\nabla_{X} h^{l}\right)(Y, Z)=\nabla_{X}^{l}\left(h^{l}(Y, Z)\right)-h^{l}\left(\nabla_{X} Y, Z\right)-h^{l}\left(Y, \nabla_{X} Z\right), \\
& \left(\nabla_{X} h^{s}\right)(Y, Z)=\nabla_{X}^{s}\left(h^{l}(Y, Z)\right)-h^{s}\left(\nabla_{X} Y, Z\right)-h^{s}\left(Y, \nabla_{X} Z\right) .
\end{aligned}
$$

Barros and Romero [1] defined indefinite Kaehler manifolds as:

Definition 2.2. Let $(\bar{M}, \bar{J}, \bar{g})$ be an indefinite almost Hermitian manifold and $\bar{\nabla}$ be the Levi-Civita connection on $\bar{M}$ with respect to $\bar{g}$. Then $\bar{M}$ is called an indefinite Kaehler manifold if $\bar{J}$ is parallel with respect to $\bar{\nabla}$, that is

$$
\left(\bar{\nabla}_{X} \bar{J}\right) Y=0, \quad \forall \quad X, Y \in \Gamma(T \bar{M}) .
$$

\section{Generalized Cauchy-Riemann lightlike submanifolds}

Definition $3.1([12])$. Let $(M, g, S(T M))$ be a real lightlike submanifold of an indefinite Kaehler manifold $(\bar{M}, \bar{g}, \bar{J})$ then $M$ is called a generalized CauchyRiemann $(G C R)$-lightlike submanifold if the following conditions are satisfied:

(A) There exist two subbundles $D_{1}$ and $D_{2}$ of $\operatorname{Rad}(T M)$ such that

$$
\operatorname{Rad}(T M)=D_{1} \oplus D_{2}, \quad \bar{J}\left(D_{1}\right)=D_{1}, \quad \bar{J}\left(D_{2}\right) \subset S(T M) .
$$

(B) There exist two subbundles $D_{0}$ and $D^{\prime}$ of $S(T M)$ such that

$$
S(T M)=\left\{\bar{J} D_{2} \oplus D^{\prime}\right\} \perp D_{0}, \quad \bar{J}\left(D_{0}\right)=D_{0}, \quad \bar{J}\left(D^{\prime}\right)=L_{1} \perp L_{2},
$$

where $D_{0}$ is a non degenerate distribution on $M, L_{1}$ and $L_{2}$ are vector subbundle of $\operatorname{ltr}(T M)$ and $S(T M)^{\perp}$, respectively.

Then the tangent bundle $T M$ of $M$ is decomposed as

$$
T M=D \perp D^{\prime}, \quad D=\operatorname{Rad}(T M) \oplus D_{0} \oplus \bar{J} D_{2} .
$$

$\mathrm{M}$ is called a proper $G C R$-lightlike submanifold if $D_{1} \neq\{0\}, D_{2} \neq\{0\}, D_{0} \neq$ $\{0\}$ and $L_{2} \neq\{0\}$.

Let $Q, P_{1}$ and $P_{2}$ be the projections on $D, \bar{J}\left(L_{1}\right)=M_{1} \subset D^{\prime}$ and $\bar{J}\left(L_{2}\right)=$ $M_{2} \subset D^{\prime}$, respectively. Then for any $X \in \Gamma(T M)$ we have

$$
X=Q X+P_{1} X+P_{2} X,
$$

applying $\bar{J}$ to (27) we obtain

$$
\bar{J} X=T X+w P_{1} X+w P_{2} X
$$


INTEGRABILITY OF DISTRIBUTIONS IN $G C R$-LIGHTLIKE SUBMANIFOLDS 595 and we can write $(28)$ as

$$
\bar{J} X=T X+w X
$$

where $T X$ and $w X$ are the tangential and transversal components of $\bar{J} X$, respectively.

Similarly

$$
\bar{J} V=B V+C V
$$

for any $V \in \Gamma(\operatorname{tr}(T M))$, where $B V$ and $C V$ are the sections of $T M$ and $\operatorname{tr}(T M)$, respectively.

Differentiating (28) and using (9)-(12) and (30) we have

$$
D^{s}\left(X, w P_{1} Y\right)=-\nabla_{X}^{s} w P_{2} Y+w P_{2} \nabla_{X} Y-h^{s}(X, T Y)+C h^{s}(X, Y),
$$

$$
D^{l}\left(X, w P_{2} Y\right)=-\nabla_{X}^{l} w P_{1} Y+w P_{1} \nabla_{X} Y-h^{l}(X, T Y)+C h^{l}(X, Y) .
$$

Using Kaehlerian property of $\bar{\nabla}$ with (11) and (12), we have the following lemmas.

Lemma 3.2. Let $M$ be a GCR-lightlike submanifold of an indefinite Kaehlerian manifold $\bar{M}$. Then we have

$$
\left(\nabla_{X} T\right) Y=A_{w Y} X+B h(X, Y)
$$

and

$$
\left(\nabla_{X}^{t} w\right) Y=C h(X, Y)-h(X, T Y),
$$

where $X, Y \in \Gamma(T M)$ and

$$
\begin{aligned}
& \left(\nabla_{X} T\right) Y=\nabla_{X} T Y-T \nabla_{X} Y, \\
& \left(\nabla_{X}^{t} w\right) Y=\nabla_{X}^{t} w Y-w \nabla_{X} Y .
\end{aligned}
$$

Lemma 3.3. Let $M$ be a GCR-lightlike submanifold of an indefinite Kaehlerian manifold $\bar{M}$. Then we have

$$
\left(\nabla_{X} B\right) V=A_{C V} X-T A_{V} X
$$

and

$$
\left(\nabla_{X}^{t} C\right) V=-w A_{V} X-h(X, B V),
$$

where $X \in \Gamma(T M), V \in \Gamma(\operatorname{tr}(T M))$ and

$$
\begin{aligned}
& \left(\nabla_{X} B\right) V=\nabla_{X} B V-B \nabla_{X}^{t} V, \\
& \left(\nabla_{X}^{t} C\right) V=\nabla_{X}^{t} C V-C \nabla_{X}^{t} V .
\end{aligned}
$$




\section{Integrability of the distributions}

Theorem 4.1. Let $M$ be a GCR-lightlike submanifold of an indefinite Kaehler manifold $\bar{M}$. If the distribution $D$ is integrable, then the following assertions hold

(i) $\bar{g}\left(D^{l}(\bar{J} X, W), Y\right)=\bar{g}\left(D^{l}(X, W), \bar{J} Y\right) \Leftrightarrow \bar{g}\left(A_{W} \bar{J} X, Y\right)=\bar{g}\left(A_{W} X, \bar{J} Y\right)$.

(ii) $\bar{g}\left(D^{l}(\bar{J} X, W), \xi\right)=\bar{g}\left(A_{W} X, \bar{J} \xi\right)$.

(iii) $\bar{g}\left(D^{l}(X, W), \xi\right)=\bar{g}\left(A_{W} \bar{J} X, \bar{J} \xi\right)$ for any $X, Y \in \Gamma(D), \xi \in \Gamma\left(D_{2}\right)$ and $W \in \Gamma\left(S\left(T M^{\perp}\right)\right)$

Proof. Let the distribution $D$ be integrable then for $X, Y \in \Gamma(D)$ and $W \in$ $\Gamma\left(S\left(T M^{\perp}\right)\right)$ we have

$$
\begin{aligned}
\bar{g}\left(D^{l}(\bar{J} X, W), Y\right) & =\bar{g}\left(\bar{\nabla}_{\bar{J} X} W+A_{W} \bar{J} X-\nabla_{\bar{J} X}^{s} W, Y\right) \\
& =-\bar{g}\left(W, \overline{\nabla_{\bar{J}}} Y\right)+\bar{g}\left(A_{W} \bar{J} X, Y\right) \\
& =-\bar{g}(W, h(\bar{J} X, Y))+\bar{g}\left(A_{W} \bar{J} X, Y\right) \\
& =-\bar{g}(W, h(X, \bar{J} Y))+\bar{g}\left(A_{W} \bar{J} X, Y\right) \\
& =-\bar{g}\left(W, \bar{\nabla}_{X} \bar{J} Y\right)+\bar{g}\left(A_{W} \bar{J} X, Y\right) \\
& =\bar{g}\left(\bar{\nabla}_{X} W, \bar{J} Y\right)+\bar{g}\left(A_{W} \bar{J} X, Y\right) \\
& =-\bar{g}\left(A_{W} X, \bar{J} Y\right)+\bar{g}\left(D^{l}(X, W), \bar{J} Y\right)+\bar{g}\left(A_{W} \bar{J} X, Y\right),
\end{aligned}
$$

or

(42) $\bar{g}\left(D^{l}(\bar{J} X, W), Y\right)-\bar{g}\left(D^{l}(X, W), \bar{J} Y\right)=\bar{g}\left(A_{W} \bar{J} X, Y\right)-\bar{g}\left(A_{W} X, \bar{J} Y\right)$.

Therefore part (i) of the assertion follows. Substituting $Y=\xi$ and $Y=\bar{J} \xi$ in (42), for any $\xi \in \Gamma\left(D_{2}\right)$, we obtain (ii) and (iii), respectively.

Theorem 4.2. Let $M$ be a GCR-lightlike submanifold of an indefinite Kaehler manifold $\bar{M}$. Then $D^{\prime}$ is integrable if and only if $\nabla_{X} \bar{J} Y=\nabla_{Y} \bar{J} X$ for any $X, Y \in \Gamma\left(D^{\prime}\right)$.

Proof. For any $X, Y \in \Gamma\left(D^{\prime}\right)$ we have

$$
h(X, \bar{J} Y)=\bar{\nabla}_{X} \bar{J} Y-\nabla_{X} \bar{J} Y
$$

and

$$
h(\bar{J} X, Y)=\bar{\nabla}_{Y} \bar{J} X-\nabla_{Y} \bar{J} X .
$$

Subtracting (44) from (43), we obtain

$$
\begin{aligned}
h(X, \bar{J} Y)-h(\bar{J} X, Y) & =\bar{J} \bar{\nabla}_{X} Y-\bar{J} \bar{\nabla}_{Y} X-\nabla_{X} \bar{J} Y+\nabla_{Y} \bar{J} X \\
& =\bar{J}[X, Y]-\nabla_{X} \bar{J} Y+\nabla_{Y} \bar{J} X \\
& =T[X, Y]+w[X, Y]-\nabla_{X} \bar{J} Y+\nabla_{Y} \bar{J} X .
\end{aligned}
$$

Equating tangential parts of above equation, we obtain

$$
T[X, Y]=\nabla_{X} \bar{J} Y-\nabla_{Y} \bar{J} X .
$$

Hence from (45) the result follows. 
Theorem 4.3. Let $M$ be a GCR-lightlike submanifold of an indefinite Kaehler manifold $\bar{M}$. Then $D_{0}$ is integrable if and only if

(i) $\bar{g}\left(h^{*}(X, Y), N\right)=\bar{g}\left(h^{*}(Y, X), N\right)$,

(ii) $\bar{g}\left(h^{*}(X, \bar{J} Y), N^{\prime}\right)=\bar{g}\left(h^{*}(Y, \bar{J} X), N^{\prime}\right)$,

(iii) $h^{s}(X, \bar{J} Y)=h^{s}(\bar{J} X, Y)$,

(iv) $\bar{g}\left(\nabla_{X}^{*} Y, \bar{J} \xi\right)=\bar{g}\left(\nabla_{Y}^{*} X, \bar{J} \xi\right)$ for any $X, Y \in \Gamma\left(D_{0}\right), N \in \Gamma(\operatorname{ltr}(T M))$, $N^{\prime} \in \Gamma\left(L_{1}\right)$ and $\xi \in \Gamma\left(D_{2}\right)$.

Proof. Using the definition of $G C R$-lightlike submanifold, $D_{0}$ is integrable if and only if

$$
\bar{g}([X, Y], N)=\bar{g}\left([X, Y], \bar{J} N^{\prime}\right)=\bar{g}([X, Y], \bar{J} W)=\bar{g}([X, Y], \bar{J} \xi)=0
$$

for any $X, Y \in \Gamma\left(D_{0}\right), N \in \Gamma(\operatorname{ltr}(T M)), N^{\prime} \in \Gamma\left(L_{1}\right), W \in \Gamma\left(L_{2}\right)$ and $\xi \in$ $\Gamma\left(D_{2}\right)$.

Using (9) and (16) we have

$$
\bar{g}([X, Y], N)=\bar{g}\left(h^{*}(X, Y), N\right)-\bar{g}\left(h^{*}(Y, X), N\right)
$$

and

$$
\begin{aligned}
\bar{g}\left([X, Y], \bar{J} N^{\prime}\right) & =\bar{g}\left(\bar{\nabla}_{X} Y, \bar{J} N^{\prime}\right)-\bar{g}\left(\bar{\nabla}_{Y} X, \bar{J} N^{\prime}\right) \\
& =-\bar{g}\left(\bar{\nabla}_{X} \bar{J} Y, N^{\prime}\right)+\bar{g}\left(\bar{\nabla}_{Y} \bar{J} X, N^{\prime}\right)
\end{aligned}
$$

using (16) we have

$$
\bar{g}\left([X, Y], \bar{J} N^{\prime}\right)=-\bar{g}\left(h^{*}(X, \bar{J} Y), N^{\prime}\right)+\bar{g}\left(h^{*}(Y, \bar{J} X), N^{\prime}\right) .
$$

Again using (9) we obtain

$$
\begin{aligned}
\bar{g}([X, Y], \bar{J} W) & =\bar{g}\left(\bar{\nabla}_{X} Y, \bar{J} W\right)-\bar{g}\left(\bar{\nabla}_{Y} X, \bar{J} W\right) \\
& =-\bar{g}\left(\bar{\nabla}_{X} \bar{J} Y, W\right)+\bar{g}\left(\bar{\nabla}_{Y} \bar{J} X, W\right) \\
& =-\bar{g}\left(h^{s}(X, \bar{J} Y), W\right)+\bar{g}\left(h^{s}(\bar{J} X, Y), W\right) .
\end{aligned}
$$

Finally from (16) we obtain

$$
\bar{g}([X, Y], \bar{J} \xi)=\bar{g}\left(\nabla_{X}^{*} Y, \bar{J} \xi\right)-\bar{g}\left(\nabla_{Y}^{*} X, \bar{J} \xi\right) .
$$

Thus from (46)-(49) the result follows.

Corollary 4.4. Let $M$ be a GCR-lightlike submanifold of an indefinite Kaehler manifold $\bar{M}$. Then $D_{0}$ is integrable if and only if

(i) $g\left(X, A_{N} Y\right)=g\left(Y, A_{N} X\right)$

(ii) $g\left(\bar{J} X, A_{N^{\prime}} Y\right)=g\left(\bar{J} Y, A_{N^{\prime}} X\right)$,

(iii) $h^{s}(X, \bar{J} Y)=h^{s}(\bar{J} X, Y)$,

(iv) $\bar{g}\left(h^{l}(X, \bar{J} Y), \xi\right)=\bar{g}\left(h^{l}(Y, \bar{J} X), \xi\right)$ for any $X, Y \in \Gamma\left(D_{0}\right), N \in \Gamma(\operatorname{ltr}(T M))$, $N^{\prime} \in \Gamma\left(L_{1}\right)$ and $\xi \in \Gamma\left(D_{2}\right)$.

Theorem 4.5. Let $M$ be a GCR-lightlike submanifold of an indefinite Kaehler manifold $\bar{M}$. Then $\operatorname{Rad}(T M)$ is integrable if and only if

(i) $\bar{g}\left(h^{l}\left(\xi, \bar{J} \xi^{\prime \prime}\right), \xi^{\prime}\right)=\bar{g}\left(h^{l}\left(\xi^{\prime}, \bar{J} \xi^{\prime \prime}\right), \xi\right)$,

(ii) $\bar{g}\left(h^{l}(\xi, Z), \xi^{\prime}\right)=\bar{g}\left(h^{l}\left(\xi^{\prime}, Z\right), \xi\right)$, 
(iii) $h^{s}\left(\xi^{\prime}, \bar{J} \xi\right)=h^{s}\left(\bar{J} \xi^{\prime}, \xi\right)$,

(iv) $\bar{g}\left(\xi, h^{l}\left(\xi^{\prime}, \bar{J} N\right)\right)=\bar{g}\left(\xi^{\prime}, h^{l}(\xi, \bar{J} N)\right)$ for any $Z \in \Gamma\left(D_{0}\right), \xi^{\prime \prime} \in \Gamma\left(D_{2}\right), \xi$, $\xi^{\prime} \in \Gamma(\operatorname{Rad}(T M)), W \in \Gamma\left(L_{2}\right)$ and $N \in \Gamma\left(L_{1}\right)$.

Proof. Using the definition of $G C R$-lightlike submanifolds, $\operatorname{Rad}(T M)$ is integrable if and only if

$$
\bar{g}\left(\left[\xi, \xi^{\prime}\right], Z\right)=\bar{g}\left(\left[\xi, \xi^{\prime}\right], \bar{J} N\right)=\bar{g}\left(\left[\xi, \xi^{\prime}\right], \bar{J} W\right)=\bar{g}\left(\left[\xi, \xi^{\prime}\right], \bar{J} \xi^{\prime \prime}\right)=0
$$

for any $Z \in \Gamma\left(D_{0}\right), \xi^{\prime \prime} \in \Gamma\left(D_{2}\right), \xi, \xi^{\prime} \in \Gamma(\operatorname{Rad}(T M)), W \in \Gamma\left(L_{2}\right)$ and $N \in$ $\Gamma\left(L_{1}\right)$.

Using (9) and (16), we obtain

$$
\begin{aligned}
\bar{g}\left(\left[\xi, \xi^{\prime}\right], \bar{J} \xi^{\prime \prime}\right) & =\bar{g}\left(\bar{\nabla}_{\xi} \xi^{\prime}, \bar{J} \xi^{\prime \prime}\right)-\bar{g}\left(\bar{\nabla}_{\xi^{\prime}} \xi, \bar{J} \xi^{\prime \prime}\right) \\
& =-\bar{g}\left(\xi^{\prime}, \bar{\nabla}_{\xi} \bar{J} \xi^{\prime \prime}\right)+\bar{g}\left(\xi, \bar{\nabla}_{\xi^{\prime}} \bar{J} \xi^{\prime \prime}\right) \\
& =-\bar{g}\left(h^{l}\left(\xi, \bar{J} \xi^{\prime \prime}\right), \xi^{\prime}\right)+\bar{g}\left(h^{l}\left(\xi^{\prime}, \bar{J} \xi^{\prime \prime}\right), \xi\right)
\end{aligned}
$$

and using (18)

$$
\begin{aligned}
\bar{g}\left(\left[\xi, \xi^{\prime}\right], Z\right) & =\bar{g}\left(\nabla_{\xi} \xi^{\prime}, Z\right)-\bar{g}\left(\nabla_{\xi^{\prime}} \xi, Z\right) \\
& =-\bar{g}\left(A_{\xi^{\prime}}^{*} \xi, Z\right)+\bar{g}\left(A_{\xi}^{*} \xi^{\prime}, Z\right) \\
& =-\bar{g}\left(h^{l}(\xi, Z), \xi^{\prime}\right)+\bar{g}\left(h^{l}\left(\xi^{\prime}, Z\right), \xi\right)
\end{aligned}
$$

and

$$
\begin{aligned}
\bar{g}\left(\left[\xi, \xi^{\prime}\right], \bar{J} W\right) & =\bar{g}\left(\bar{\nabla}_{\xi} \xi^{\prime}, \bar{J} W\right)-\bar{g}\left(\bar{\nabla}_{\xi^{\prime}} \xi, \bar{J} W\right) \\
& =-\bar{g}\left(W, \bar{\nabla}_{\xi} \bar{J} \xi^{\prime}\right)+\bar{g}\left(W, \bar{\nabla}_{\xi^{\prime}} \bar{J} \xi\right) \\
& =-\bar{g}\left(h^{s}\left(\xi, \bar{J} \xi^{\prime}\right), W\right)+\bar{g}\left(h^{s}\left(\xi^{\prime}, \bar{J} \xi\right), W\right)
\end{aligned}
$$

and

$$
\begin{aligned}
\bar{g}\left(\left[\xi, \xi^{\prime}\right], \bar{J} N\right) & =\bar{g}\left(\bar{\nabla}_{\xi} \xi^{\prime}, \bar{J} N\right)-\bar{g}\left(\bar{\nabla}_{\xi^{\prime}} \xi, \bar{J} N\right) \\
& =-\bar{g}\left(\xi^{\prime}, \bar{\nabla}_{\xi} \bar{J} N\right)+\bar{g}\left(\xi, \bar{\nabla}_{\xi^{\prime}} \bar{J} N\right) \\
& =-\bar{g}\left(\xi^{\prime}, h^{l}(\xi, \bar{J} N)\right)+\bar{g}\left(\xi, h^{l}\left(\xi^{\prime}, \bar{J} N\right)\right) .
\end{aligned}
$$

Thus from (50)-(53), the result follows.

Corollary 4.6. Let $M$ be a GCR-lightlike submanifold of an indefinite Kaehler manifold $\bar{M}$. Then $\operatorname{Rad}(T M)$ is integrable if and only if

(i) $A_{\xi}^{*} \xi^{\prime} \notin \Gamma\left(M_{1}\right)$,

(ii) $g\left(\nabla_{\xi^{\prime}}^{*} \bar{J} Z, \bar{J} \xi\right)=g\left(\nabla_{\xi}^{*} \bar{J} Z, \bar{J} \xi^{\prime}\right)$,

(iii) $g\left(A_{W} \xi^{\prime}, \bar{J} \xi\right)=g\left(A_{W} \xi, \bar{J} \xi^{\prime}\right)$,

(iv) $g\left(A_{N} \xi^{\prime}, \bar{J} \xi\right)=g\left(A_{N} \xi, \bar{J} \xi^{\prime}\right)$ for any $Z \in \Gamma\left(D_{0}\right), \xi, \xi^{\prime} \in \Gamma(\operatorname{Rad}(T M))$ and $N \in \Gamma(\operatorname{ltr}(T M))$.

Theorem 4.7. Let $M$ be a GCR-lightlike submanifold of an indefinite Kaehler manifold $\bar{M}$. Then the distribution $D_{1}$ is integrable if and only if

(i) $\nabla_{X}^{* t} \bar{J} Y-\nabla_{Y}^{* t} \bar{J} X \in \Gamma\left(D_{1}\right)$,

(ii) $A_{\bar{J} Y}^{*} X=A_{\bar{J} X}^{*} Y$, 
(iii) $B h(X, \bar{J} Y)=B h(Y, \bar{J} X)$ for any $X, Y \in \Gamma\left(D_{1}\right)$.

Proof. Since $\bar{J}$ is the almost complex structure on $M$ therefore for any $X, Y \in$ $\Gamma\left(D_{1}\right)$ we have

$$
\bar{\nabla}_{X} Y=-\bar{\nabla}_{X} \bar{J}^{2} Y=-\bar{J} \bar{\nabla}_{X} \bar{J} Y
$$

using (9), we have

$$
\nabla_{X} Y+h(X, Y)=-\bar{J}\left(\nabla_{X} \bar{J} Y+h(X, \bar{J} Y)\right) .
$$

Now, using (17), (29)-(30) and then equating the tangential components of the resulting equation both sides we obtain

$$
\nabla_{X} Y=T A_{\bar{J} Y}^{*} X-T \nabla_{X}^{* t} \bar{J} Y-B h(X, \bar{J} Y),
$$

replacing $X$ by $Y$ and then subtracting the resulting equation from (54) we obtain

$(55)$

$[X, Y]=T\left(A_{\bar{J} Y}^{*} X-A_{\bar{J} X}^{*} Y\right)-T\left(\nabla_{X}^{* t} \bar{J} Y-\nabla_{Y}^{* t} \bar{J} X\right)-B h(X, \bar{J} Y)+B h(Y, \bar{J} X)$.

Hence from (55) the result follows.

Corollary 4.8. Let $M$ be a GCR-lightlike submanifold of an indefinite Kaehler manifold $\bar{M}$. Then $D_{1}$ defines a totally geodesic foliation in $M$ if and only if

(i) $\nabla_{X}^{* t} \bar{J} Y \in \Gamma\left(D_{1}\right)$,

(ii) $A_{\bar{J} Y}^{*} X=0$,

(iii) $B h(X, \bar{J} Y)=0$ for any $X, Y \in \Gamma\left(D_{1}\right)$.

Theorem 4.9. Let $M$ be a GCR-lightlike submanifold of an indefinite Kaehler manifold $\bar{M}$. Then the distribution $D_{2}$ is integrable if and only if

(i) $\nabla_{X}^{*} \bar{J} Y-\nabla_{Y}^{*} \bar{J} X \in \Gamma\left(\bar{J} D_{2}\right)$,

(ii) $h^{*}(X, \bar{J} Y)=h^{*}(Y, \bar{J} X)$,

(iii) $B h(X, \bar{J} Y)=B h(Y, \bar{J} X)$ for any $X, Y \in \Gamma\left(D_{2}\right)$.

Proof. Since $\bar{J}$ is the almost complex structure on $M$ therefore for any $X, Y \in$ $\Gamma\left(D_{2}\right)$ we have

$$
\nabla_{X} Y+h(X, Y)=-\bar{J}\left(\nabla_{X} \bar{J} Y+h(X, \bar{J} Y)\right)
$$

using (16) and (29)-(30) and then equating tangential components of the resulting equation both sides we obtain

$$
\nabla_{X} Y=-T \nabla_{X}^{*} \bar{J} Y-T h^{*}(X, \bar{J} Y)-B h(X, \bar{J} Y),
$$

replacing $X$ and $Y$ and then subtracting the resulting equation from (56) we obtain

$$
\begin{aligned}
{[X, Y]=} & -T\left(\nabla_{X}^{*} \bar{J} Y-\nabla_{Y}^{*} \bar{J} X\right)-T\left(h^{*}(X, \bar{J} Y)-h^{*}(Y, \bar{J} X)\right) \\
& -B h(X, \bar{J} Y)+B h(Y, \bar{J} X) .
\end{aligned}
$$

Hence from (57) the theorem follows. 
Corollary 4.10. Let $M$ be a GCR-lightlike submanifold of an indefinite Kaehler manifold $\bar{M}$. Then the distribution $D_{2}$ defines a totally geodesic foliation in $M$ if and only if

(i) $\nabla_{X}^{*} \bar{J} Y \in \Gamma\left(\bar{J} D_{2}\right)$,

(ii) $h^{*}(X, \bar{J} Y)=0$,

(iii) $B h(X, \bar{J} Y)=0$ for any $X, Y \in \Gamma\left(D_{2}\right)$.

Theorem 4.11. Let $M$ be a GCR-lightlike submanifold of an indefinite Kaehler manifold $\bar{M}$. Then $\bar{J} D_{2}$ is integrable if and only if

(i) $\bar{g}\left(\bar{J} \xi, A_{N} \bar{J} \xi^{\prime}\right)=\bar{g}\left(\bar{J} \xi^{\prime}, A_{N} \bar{J} \xi\right)$,

(ii) $h^{s}\left(\bar{J} \xi, \xi^{\prime}\right)=h^{s}\left(\bar{J} \xi^{\prime}, \xi\right)$,

(iii) $\bar{g}\left(h^{l}\left(\bar{J} \xi, \xi^{\prime}\right), \xi^{\prime \prime}\right)=\bar{g}\left(h^{l}\left(\xi, \bar{J} \xi^{\prime}\right), \xi^{\prime \prime}\right)$,

(iv) $\bar{g}\left(\bar{J} Z, A_{\xi}^{*} \bar{J} \xi^{\prime}\right)=\bar{g}\left(\bar{J} Z, A_{\xi^{\prime}}^{*} \bar{J} \xi\right)$ for any $Z \in \Gamma\left(D_{0}\right), \xi, \xi^{\prime}, \xi^{\prime \prime} \in \Gamma\left(D_{2}\right)$, $W \in \Gamma\left(L_{2}\right)$ and $N \in \Gamma(\operatorname{ltr}(T M))$.

Proof. Using the definition of $G C R$-lightlike submanifolds, $\bar{J} D_{2}$ is integrable if and only if

$$
\bar{g}\left(\left[\bar{J} \xi^{\prime}, \bar{J} \xi\right], N\right)=\bar{g}\left(\left[\bar{J} \xi^{\prime}, \bar{J} \xi\right], \bar{J} W\right)=\bar{g}\left(\left[\bar{J} \xi^{\prime}, \bar{J} \xi\right], \bar{J} \xi^{\prime \prime}\right)=\bar{g}\left(\left[\bar{J} \xi^{\prime}, \bar{J} \xi\right], Z\right)=0
$$

for any $Z \in \Gamma\left(D_{0}\right), \xi, \xi^{\prime}, \xi^{\prime \prime} \in \Gamma\left(D_{2}\right), W \in \Gamma\left(L_{2}\right)$ and $N \in \Gamma(\operatorname{ltr}(T M))$.

Using (11) we have

$$
\begin{aligned}
\bar{g}\left(\left[\bar{J} \xi^{\prime}, \bar{J} \xi\right], N\right) & =\bar{g}\left(\bar{\nabla}_{\bar{J} \xi^{\prime}} \bar{J} \xi, N\right)-\bar{g}\left(\bar{\nabla}_{\bar{J} \xi} \bar{J} \xi^{\prime}, N\right) \\
& =-\bar{g}\left(\bar{J} \xi, \bar{\nabla}_{\bar{J} \xi^{\prime}} N\right)+\bar{g}\left(\bar{J} \xi^{\prime}, \bar{\nabla}_{\bar{J} \xi} N\right) \\
& =\bar{g}\left(\bar{J} \xi, A_{N} \bar{J} \xi^{\prime}\right)-\bar{g}\left(\bar{J} \xi^{\prime}, A_{N} \bar{J} \xi\right)
\end{aligned}
$$

and

$$
\begin{aligned}
\bar{g}\left(\left[\bar{J} \xi^{\prime}, \bar{J} \xi\right], \bar{J} W\right) & =\bar{g}\left(\bar{\nabla}_{\bar{J} \xi^{\prime}} \bar{J} \xi, \bar{J} W\right)-\bar{g}\left(\bar{\nabla}_{\bar{J} \xi} \bar{J} \xi^{\prime}, \bar{J} W\right) \\
& =\bar{g}\left(\bar{\nabla}_{\bar{J} \xi^{\prime}} \xi, W\right)-\bar{g}\left(\bar{\nabla}_{\bar{J} \xi} \xi^{\prime}, W\right) \\
& =\bar{g}\left(h^{s}\left(\bar{J} \xi^{\prime}, \xi\right), W\right)-\bar{g}\left(h^{s}\left(\bar{J} \xi, \xi^{\prime}\right), W\right)
\end{aligned}
$$

and

$$
\begin{aligned}
\bar{g}\left(\left[\bar{J} \xi^{\prime}, \bar{J} \xi\right], \bar{J} \xi^{\prime \prime}\right) & =\bar{g}\left(\bar{\nabla}_{\bar{J} \xi^{\prime}} \bar{J} \xi, \bar{J} \xi^{\prime \prime}\right)-\bar{g}\left(\bar{\nabla}_{\bar{J} \xi} \bar{J} \xi^{\prime}, \bar{J} \xi^{\prime \prime}\right) \\
& =\bar{g}\left(\bar{\nabla}_{\bar{J} \xi^{\prime}} \xi, \xi^{\prime \prime}\right)-\bar{g}\left(\bar{\nabla}_{\bar{J} \xi} \xi^{\prime}, \xi^{\prime \prime}\right) \\
& =\bar{g}\left(h^{l}\left(\bar{J} \xi^{\prime}, \xi\right), \xi^{\prime \prime}\right)-\bar{g}\left(h^{l}\left(\bar{J} \xi, \xi^{\prime}\right), \xi^{\prime \prime}\right)
\end{aligned}
$$

and finally

$$
\begin{aligned}
\bar{g}\left(\left[\bar{J} \xi^{\prime}, \bar{J} \xi\right], Z\right) & =\bar{g}\left(\bar{\nabla}_{\bar{J} \xi^{\prime}} \bar{J} \xi, Z\right)-\bar{g}\left(\bar{\nabla}_{\bar{J} \xi} \bar{J} \xi^{\prime}, Z\right) \\
& =-\bar{g}\left(\bar{\nabla}_{\bar{J} \xi^{\prime}} \xi, \bar{J} Z\right)+\bar{g}\left(\bar{\nabla}_{\bar{J} \xi} \xi^{\prime}, \bar{J} Z\right) \\
& =\bar{g}\left(\bar{J} Z, A_{\xi}^{*} \bar{J} \xi^{\prime}\right)-\bar{g}\left(\bar{J} Z, A_{\xi^{\prime}}^{*} \bar{J} \xi\right) .
\end{aligned}
$$

Hence from (58)-(61) the result follows. 
Lemma 4.12. Let $M$ be a GCR-lightlike submanifold of an indefinite Kaehler manifold $\bar{M}$. If $M$ is D-geodesic, then $D$ defines a totally geodesic foliation in $M$.

Proof. For $X, Y \in \Gamma(D)$, using (31) and (32), we obtain $w \nabla_{X} Y=h(X, T Y)-$ $C h(X, Y)$, then using the hypothesis, we obtain $w \nabla_{X} Y=0$. Thus the proof is complete.

Lemma 4.13. Let $M$ be a GCR-lightlike submanifold of an indefinite Kaehler manifold $\bar{M}$. If $T$ is parallel, then $B h(X, Y)=0$ for any $Y \in \Gamma(D)$.

Proof. For any $X, Y \in \Gamma(T M)$, from (33) we have

$$
\left(\nabla_{X} T\right) Y=A_{w Y} X+B h(X, Y)
$$

From the hypothesis of lemma, the proof is complete.

Theorem 4.14 ([12]). Let $M$ be a GCR-lightlike submanifold of an indefinite Kaehler manifold $\bar{M}$. Then the distribution $D$ defines a totally geodesic foliation in $M$ if and only if $B h(X, Y)=0 \forall X, Y \in \Gamma(D)$.

Theorem 4.15. Let $M$ be a GCR-lightlike submanifold of an indefinite Kaehler manifold $\bar{M}$. If $T$ is parallel, then the distribution $D$ defines a totally geodesic foliation in $M$.

Proof. Let $T$ is parallel, then from Lemma 4.13, we obtain $B h(X, Y)=0$ for any $X \in \Gamma(T M)$ and $Y \in \Gamma(D)$. Then using Theorem 4.14, D defines a totally geodesic foliation in $M$.

Theorem 4.16. Let $M$ be a GCR-lightlike submanifold of an indefinite Kaehler manifold $\bar{M}$. Then the distribution $D^{\prime}$ is parallel if and only if $A_{\bar{J} Y} X$ has no components in holomorphic distribution for any $X, Y \in \Gamma\left(D^{\prime}\right)$.

Proof. From (33), we have

$$
-T \nabla_{X} Y=A_{w Y} X+B h(X, Y)
$$

for any $X, Y \in \Gamma\left(D^{\prime}\right)$. Hence the proof follows.

\section{References}

[1] M. Barros and A. Romero, Indefinite Kähler manifolds, Math. Ann. 261 (1982), no. 1, 55-62.

[2] A. Bejancu, CR submanifolds of a Kaehler manifold I, Proc. Amer. Math. Soc. 69 (1978), no. 1, 135-142.

[3] _ CR submanifolds of a Kaehler manifold II, Trans. Amer. Math. Soc. 250 (1979), $333-345$.

[4] A. Bejancu, M. Kon, and K. Yano, CR submanifolds of a complex space form, J. Differential Geom. 16 (1981), no. 1, 137-145.

[5] D. E. Blair and B. Y. Chen, On CR submanifolds of Hermitian manifolds, Israel J. Math. 34 (1979), no. 4, 353-363.

[6] B. Y. Chen, CR submanifolds of a Kaehler manifold I, J. Differential Geom. 16 (1981), no. 2, 305-322. 
[7] K. L. Duggal, CR-structures and Lorentzian geometry, Acta Appl. Math. 7 (1986), no. $3,211-223$.

[8] L Lorentzian geometry of CR submanifolds, Acta Appl. Math. 17 (1989), no. 2, $171-193$

[9] K. L. Duggal and A. Bejancu, Lightlike Submanifolds of Semi-Riemannian Manifolds and Applications, Vol. 364 of Mathematics and its Applications, Kluwer Academic Publishers, Dordrecht, The Netherlands, 1996.

[10] K. L. Duggal and D. H. Jin, Totally umbilical lightlike submanifolds, Kodai Math. J. 26 (2003), no. 1, 49-68.

[11] K. L. Duggal and B. Sahin, Screen Cauchy-Riemann lightlike submanifolds, Acta Math. Hungar. 106 (2005), no. 1-2, 137-165.

[12] — Generalized Cauchy-Riemann lightlike submanifolds of Kaehler manifolds, Acta Math. Hungar. 112 (2006), no. 1-2, 107-130.

[13] Kumar, Rakesh, Rani, Rachna, and R. K. Nagaich, Some properties of lightlike submanifolds of semi-Riemannian manifolds, Demonstratio Math. 43 (2010), no. 3, 691-701.

[14] B. Sahin and R. Günes, Integrability of distributions in CR-lightlike submanifolds, Tamkang J. Math. 33 (2002), no. 3, 209-221.

[15] — Geodesic CR-Lightlike submanifolds, Beiträge Algebra Geom. 42 (2001), no. 2, $583-594$.

[16] R. Sharma and K. L. Duggal, Mixed foliate CR submanifolds of indefinite complex space forms, Ann. Mat. Pura Appl. (4) 149 (1987), 103-111.

[17] K. Yano and M. Kon, Differential geometry of CR submanifolds, Geom. Dedicata 10 (1981), no. 1-4, 369-391.

[18] — CR Submanifolds of Kaehlerian and Sasakian Manifolds, Birkhauser, Bostan, 1983.

RAKeSh KumAR

University COLLEge of Engineering

PunJabi University

Patiala 147002, India

E-mail address: rakesh-ucoe@pbi.ac.in

SANGeET KUMAR

Rayat Institute of Engineering \& Information Technology

RAILMAJRA, SBS NAGAR 144533, INDIA

E-mail address: sp7maths@gmail.com

Rakesh Kumar Nagaich

Department of Mathematics

Punjabi University

PATiala 147002, India

E-mail address: nagaichrakesh@yahoo.com 